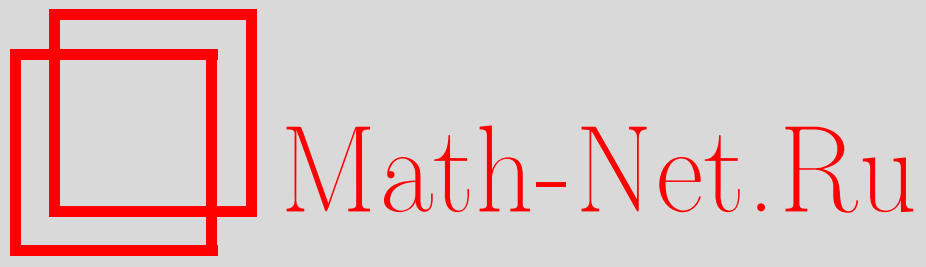

С. А. Назаров, Эллиптические краевые задачи на гибридных областях, Функи. анализ и его прил., 2004, том 38, выпуск 4, 55-72

DOI: https://doi.org/10.4213/faa126

Использование Общероссийского математического портала MathNet.Ru подразумевает, что вы прочитали и согласны с пользовательским соглашением

http://www . mathnet.ru/rus/agreement

Параметры загрузки:

IP : 54.198 .67 .100

26 апреля 2023 г., 15:09:51

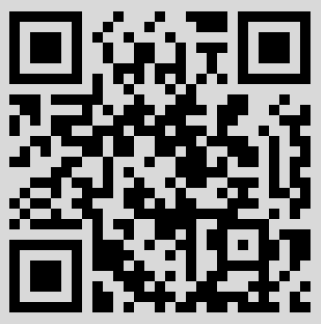


Функииональньй анализ и его приложения

2004, т. 38 , вып. 4, с. 55-72

УДК 517.923

\title{
Эллиптические краевые задачи на гибридных областях
}

\author{
(C) 2004. C. A. НАзАРов
}

Посвящается Виктору Борисовичу Лидскому
в связи с его юбилеем

\section{§1. Введение}

На гладкой границе $\partial \Omega$ области $\Omega \subset \mathbb{R}^{3}$ с компактным замыканием $\bar{\Omega}$ выберем точки $P^{1}, \ldots, P^{J}$, из которых наружу выпустим попарно непересекающиеся лучи $\Lambda^{j}, j=1, \ldots, J$. Обозначим через $z^{j} \in \mathbb{R}_{+}$координату на $\Lambda^{j}$ и отсечем интервалы $\Upsilon^{j}=\left\{x: z^{j} \in\left(0, l_{j}\right)\right\} \subset \Lambda^{j}$ с длинами $l_{j}>0$. Основной целью статьи является описание корректной постановки на множестве

$$
\Omega^{\star}=\Omega \cup\left(\Upsilon^{1} \cup \cdots \cup \Upsilon^{J}\right)
$$

(«кактус с одномерными иглами») эллиптической задачи с условиями сопряжения (трансмиссии) в точках $P^{1}, \ldots, P^{J}$. Подразумевается, что эта задача должна оказаться формально самосопряженной и допускать вариационную постановку.

Краевые задачи на «гибридных» множествах, составленных из стратов различных размерностей, рассматривались в публикациях [1-6] и др., но из-за отсутствия в обсуждаемом здесь случае необходимых следовых теорем полученные ранее результаты не удается приспособить к сочленению (1.1) - подходы, опирающиеся на структурированные энергетические функционалы, стратифицированные меры и пр., приводят к распадению задачи в $\Omega^{\star}$ на зачастую нестандартные, но всегда решаемые по отдельности задачи для тела $\Omega$ и для отрезков $\Upsilon^{j}$.

В данной статье предлагается новый подход, основанный на применении обобщенных формул Грина (см. $[7,8]$ и др.) в пространствах функций с предписанным асимптотическим поведением около точек $P^{1}, \ldots, P^{J}$ (предложение 3.1 и лемма 4.1). Эти формулы позволяют так определить условия сопряжения на $\mathbf{P}=\left\{P^{1}, \ldots, P^{J}\right\}$, связывающие коэффициенты асимптотических разложений, что полученная задача $(\star)$ на $\Omega^{\star}$ (см. $\left.\S 4\right)$ приобретает все свойства обычных самосопряженных эллиптических краевых задач, в частности, нулевой индекс (предложение 4.2) и энергетический функционал, стационарными точками которого являются решения задачи $(\star)$ и только они (предложение 5.1). При подходящем строении матричных дифференциальных операторов условия сопряжения в точках $P^{1}, \ldots, P^{J}$ дают возможность сопоставить сформулированной задаче на $\Omega^{\star}$ самосопряженное расширение некоторого оператора в векторном пространстве $L_{2}\left(\Omega^{\star}\right)^{n}$ с областью определения $C_{0}^{\infty}\left(\Omega^{\star}\right)^{n}$ (предложение 6.1).

* Работа выполнена при финансовой поддержке Российского фонда фундаментальных исследований (проект 03-01-00835). 
В §7, где речь идет о моделировании сочленений тонких и массивных тел, результаты асимптотического анализа (см. [9-11]) интерпретируются с позиций постановки условий сопряжения.

Финальные формулы, но не сам ход рассуждений, весьма чувствительны к порядкам дифференциальных операторов и размерностям областей, составляющих гибридное множество. Во избежание нагромождения индексов ограничимся рассмотрением системы уравнений второго порядка на трехмерном теле $\Omega$. Предполагается, что эта система обладает полиномиальным свойством [12], а строение систем обыкновенных дифференциальных уравнений на отрезках $\Upsilon^{j}$ соотносится с соответствующим подпространством векторных полиномов (см. комментарии к формуле (2.8) и ср. с замечанием 7.2).

\section{§2. Эллиптические системы с полиномиальным свойством}

Рассмотрим $n \times n$-матрицу дифференциальных операторов

$$
L^{0}\left(x, \nabla_{x}\right)=D^{0}\left(-\nabla_{x}\right)^{\top} A^{0}(x) D^{0}\left(\nabla_{x}\right),
$$

где ${ }^{\top}$ - знак транспонирования, $A$ - симметрическая и положительно определенная $m \times m$-матрица-функция с вещественными элементами из $C^{\infty}(\bar{\Omega})$, а $D^{0}\left(\nabla_{x}\right)$ - матрица размера $m \times n$, составленная из дифференциальных операторов первого порядка и являющаяся алгебраически комплектной [13], т. е. при каком-то $\rho_{D} \in \mathbb{N}:=\{1,2, \ldots\}$ для любой строки $q(\xi)=\left(q_{1}(\xi), \ldots, q_{n}(\xi)\right)$ однородных полиномов степени $\rho \geqslant \rho_{D}$ найдется полиномиальная строка $Q(\xi)=$ $\left(Q_{1}(\xi), \ldots, Q_{m}(\xi)\right)$, удовлетворяющая равенству

$$
q(\xi)=Q(\xi) D^{0}(\xi) \quad \forall \xi \in \mathbb{R}^{3} .
$$

Согласно [12], оператор (2.1) обладает полиномиальным свойством в полном обгеме:

$$
u \in C^{\infty}(\bar{\Xi})^{n}, a^{0}(u, u ; \Xi)=\left.0 \Longleftrightarrow u \in \mathscr{P}\right|_{\Xi}
$$

Здесь $\Xi-$ произвольная область в $\mathbb{R}^{3}, \mathscr{P}$ - линеал векторных полиномов, причем

$$
\mathscr{P}=\left\{p=\left(p_{1}, \ldots, p_{n}\right)^{\top}: D^{0}\left(\nabla_{x}\right) p(x)=0, x \in \mathbb{R}^{3}\right\}
$$

и его конечномерность вытекает из требования (2.2) (см. [12]), а $a^{0}$ - симметрическая и неотрицательная квадратичная форма, фигурирующая в формуле Грина

$$
\left(L^{0} u, v\right)_{\Omega}+\left(N^{0} u, v\right)_{\partial \Omega}=a^{0}(u, v ; \Omega):=\left(A^{0} D^{0}\left(\nabla_{x}\right) u, D^{0}\left(\nabla_{x}\right) v\right)_{\Omega},
$$

в которой $N^{0}\left(x, \nabla_{x}\right)=D^{0}(\nu(x))^{\top} A^{0}(x) D^{0}\left(\nabla_{x}\right)$ - оператор краевых условий Неймана, $\nu$ - единичный вектор внешней нормали к поверхности $\partial \Omega,(,)_{\Omega}$ - скалярное произведение в пространстве $L_{2}(\Omega)^{m}$ (вне зависимости от размерности $m)$.

На теле $\Omega$ рассматриваем краевую задачу Неймана

$$
\begin{aligned}
L^{0}\left(x, \nabla_{x}\right) u^{0}(x) & =f^{0}(x), & & x \in \Omega, \\
N^{0}\left(x, \nabla_{x}\right) u^{0}(x) & =g^{0}(x), & & x \in \partial \Omega \backslash \mathbf{P},
\end{aligned}
$$

где $u^{0}=\left(u_{1}^{0}, \ldots, u_{J}^{0}\right)^{\top}$ и

$$
f^{0}=\left(f_{1}^{0}, \ldots, f_{J}^{0}\right)^{\top} \in L_{2}(\Omega)^{n}, \quad g^{0}=\left(g_{1}^{0}, \ldots, g_{J}^{0}\right)^{\top} \in H^{1 / 2}(\partial \Omega)^{n} .
$$


При этом применяются стандартные обозначения пространств Соболева-Слободецкого. Для описания структуры подходящих матричных операторов $L^{j}\left(z^{j}, \partial / \partial z^{j}\right)$ из систем обыкновенных дифференциальных уравнений на отpeзках

$$
L^{j}\left(z^{j}, \partial_{z^{j}}\right) u^{j}\left(z^{j}\right)=f^{j}\left(z^{j}\right), \quad z^{j} \in \Upsilon^{j},
$$

потребуются дополнительные построения. В силу (2.4) производные любого векторного полинома $p \in \mathscr{P}$ также принадлежат $\mathscr{P}$. Поэтому в линеале $\mathscr{P}$ можно ввести базис $\left\{p_{(j)}^{k, i}: k=1, \ldots, T^{(j)}, i=0, \ldots, t_{k}^{(j)}-1\right\}$, соотнесенный с направлением луча $\Lambda^{j}$ и обладающий следующими свойствами:

$1^{\circ}$ полиномь $p_{(j)}^{1,0}, \ldots, p_{(j)}^{T^{(j)}, 0}$ не зависят от переменной $z^{j}$

$2^{\circ}$ при $k=1, \ldots, T^{(j)}$ u $i=1, \ldots, t_{k}^{(j)}-1$ выполняются равенства $\partial_{z^{j}} p_{(j)}^{k, i}=$ $p_{(j)}^{k, i-1}$.

Ясно, что $t_{1}^{(j)}+\cdots+t_{T^{(j)}}^{(j)}=\operatorname{dim} \mathscr{P}:=d_{0}$. Только для устранения индекса $j$ в обозначениях $t_{k}^{(j)}$ и $T^{(j)}$ предположим, что эти числа не зависят от направления $z^{j}$ (свойство изотропии линеала $\mathscr{P}$ ). В качестве $L^{j}$ возьмем матрицы дифференциальных операторов размера $T \times T$,

$$
L^{j}\left(z^{j}, \partial_{z^{j}}\right)=\mathscr{D}\left(-\partial_{z^{j}}\right)^{\top} A^{j}\left(z^{j}\right) \mathscr{D}\left(\partial_{z^{j}}\right) ;
$$

здесь $A^{j}$ - симметрическая и положительно определенная $T \times T$-матрицафункция с элементами из $C^{\infty}\left[0, l_{j}\right]$, а $\mathscr{D}\left(\partial_{z}\right)$ - диагональная матрица $\operatorname{diag}\left\{\partial_{z}^{t_{1}}, \ldots, \partial_{z}^{t_{T}}\right\}$ (символ ${ }^{\top}$ в $(2.9)$ можно не писать). Опять-таки для упрощения изложения назначим в точках $z^{j}=l_{j}$ граничные условия Дирихле

$$
D^{j}\left(\partial_{z^{j}}\right) u^{j}\left(l_{j}\right)=0,
$$

причем матрица $D^{j}$ (алгебраически комплектная) имеет размер $d_{0} \times T$, а левая часть равенства (2.10) выглядит так:

$$
\left(u_{1}^{j}\left(l_{j}\right), \partial_{z^{j}} u_{1}^{j}\left(l_{j}\right), \ldots, \partial_{z^{j}}^{t_{1}-1} u_{1}^{j}\left(l_{j}\right), \ldots, u_{T}^{j}\left(l_{j}\right), \partial_{z^{j}} u_{T}^{j}\left(l_{j}\right), \ldots, \partial_{z^{j}}^{t_{T}-1} u_{T}^{j}\left(l_{j}\right)\right)^{\top} .
$$

ПримеР 2.1. 1) Если $\mathscr{P}=\mathbb{R}^{n}$ (в частности, $L^{0}$ - скалярный оператор в дивергентной форме и $\left.D^{0}\left(\nabla_{x}\right)=\nabla_{x}\right)$, то $T=n$ и $t_{1}=\cdots=t_{T}=1$, а значит, $L^{j}$ также состоит из операторов второго порядка.

2) Для оператора $L^{0}$ трехмерной системы теории упругости, анизотропной и неоднородной, $\mathscr{P}=\left\{p(x)=\mathbf{p}(x) a: a \in \mathbb{R}^{6}\right\}$ - линеал жестких смещений, инвариантный относительно ортогональных преобразований координат. Матрицы $\mathbf{p}(x)$ и $D^{0}(x)^{\top}$ размера $3 \times 6$ имеют соответственно вид

$$
\left(\begin{array}{cccccc}
1 & 0 & 0 & 0 & -\alpha x_{3} & \alpha x_{2} \\
0 & 1 & 0 & \alpha x_{3} & 0 & -\alpha x_{1} \\
0 & 0 & 1 & -\alpha x_{2} & \alpha x_{1} & 0
\end{array}\right), \quad\left(\begin{array}{cccccc}
x_{1} & 0 & 0 & 0 & \alpha x_{3} & \alpha x_{2} \\
0 & x_{2} & 0 & \alpha x_{3} & 0 & \alpha x_{1} \\
0 & 0 & x_{3} & \alpha x_{2} & \alpha x_{1} & 0
\end{array}\right),
$$

где $\alpha=2^{-1 / 2}$ - нормирующий множитель. Любую линейную вектор-функцию можно разложить по столбцам этих матриц, а $D^{0}(\xi)$ удовлетворяет условию (2.2) с $\rho_{D}=2$. Нетрудно убедиться в том, что $n=3, T=4$ и $t_{1}=t_{2}=2$, $t_{3}=t_{4}=1$ (см. примеры 1.12 и 4.3(1) в [12]), причем в изотропном случае физически оправдан выбор диагональной матрицы $A^{j}-$ система (2.8) распадается на четыре независимых уравнения с порядками 4, 4 и 2,2 (см. [14]). 
Предположим, что в уравнении (2.8)

$$
f^{j}=\left(f_{1}^{j}, \ldots, f_{T}^{j}\right)^{\top} \in \mathscr{H}^{-\mathbf{t}}\left(\Upsilon^{j}\right)=\prod_{k=1}^{T} H^{-t_{k}+\max \mathbf{t}}\left(\Upsilon^{j}\right),
$$

где $\max \mathbf{t}=\max \left\{t_{1}, \ldots, t_{T}\right\}$. Таким образом, решение $u^{j}=\left(u_{1}^{j}, \ldots, u_{T}^{j}\right)^{\top}$ логично искать в подпространстве

$$
\dot{\mathscr{H}}^{\mathbf{t}}\left(\Upsilon^{j}\right)=\left\{u^{j} \in \prod_{k=1}^{T} H^{t_{k}+\max \mathbf{t}}\left(\Upsilon^{j}\right): u^{j} \text { удовлетворяет равенству (2.10) }\right\} .
$$

Из вектор-функций, фигурирующих в (2.6) и в $(2.8),(2.9)$, составим столбец

$$
\mathbf{u}=\left(u^{0}, u^{1}, \ldots, u^{J}\right)^{\top} \in \mathbf{H}:=H^{2}(\Omega)^{n} \times \dot{\mathscr{H}}^{\mathbf{t}}\left(\Upsilon^{1}\right) \times \cdots \times \dot{\mathscr{H}}^{\mathbf{t}}\left(\Upsilon^{J}\right) .
$$

Если $\mathbf{u}, \mathbf{v} \in \mathbf{H}$, то справедливы формулы Грина

$$
\begin{aligned}
& \left(L^{0} u^{0}, v^{0}\right)_{\Omega}+\left(N^{0} u^{0}, v^{0}\right)_{\partial \Omega}=\left(u^{0}, L^{0} v^{0}\right)_{\Omega}+\left(u^{0}, N^{0} u^{0}\right)_{\partial \Omega}, \\
& \left(L^{j} u^{j}, v^{j}\right)_{\Upsilon^{j}}+\left\langle N^{j}\left(\partial_{z^{j}}\right) u^{j}(0), D^{j}\left(\partial_{z^{j}}\right) v^{j}(0)\right\rangle \\
& =\left(u^{j}, L^{j} v^{j}\right)_{\Upsilon^{j}}+\left\langle D^{j}\left(\partial_{z^{j}}\right) u^{j}(0), N^{j}\left(\partial_{z^{j}}\right) v^{j}(0)\right\rangle,
\end{aligned}
$$

где $N^{j}\left(\partial_{z^{j}}\right)$ - оператор граничных условий Неймана для системы (2.8) в точке $z^{j}=0$, а через $\langle$,$\rangle обозначено скалярное произведение в \mathbb{R}^{m}$ (вне зависимости от размерности $m$ ).

\section{§3. Сингулярные решения задачи в $\Omega$}

При $l \in \mathbb{N}_{0}=\mathbb{N} \cup\{0\}$ и $\beta \in \mathbb{R}$ введем пространство $V_{\beta}^{l}(\Omega)$ как пополнение линеала $C_{0}^{\infty}(\bar{\Omega} \backslash \mathbf{P})$ по весовой норме

$$
\left\|v ; V_{\beta}^{l}(\Omega)\right\|=\left(\sum_{q=0}^{l}\left\|\mathfrak{r}^{\beta-l+q} \nabla_{x}^{q} v ; L_{2}(\Omega)\right\|^{2}\right)^{1 / 2},
$$

где $\nabla_{x}^{q}$ - совокупность производных функции $v$ порядка $q$, a $\mathfrak{r} \in C^{\infty}(\bar{\Omega} \backslash \mathbf{P})-$ весовая функция, положительная всюду в $\bar{\Omega}$, кроме точек $P^{j}$, и совпадающая с $r_{j}=\left|x-P^{j}\right|$ в окрестностях этих точек. Иными словами, в $V_{\beta}^{l}(\Omega)$ включены те функции $v \in H_{\text {loc }}^{l}(\bar{\Omega} \backslash \mathbf{P})^{n}$, для которых конечна норма (3.1). При $l \geqslant 1$ под $V_{\beta}^{l-1}(\partial \Omega)$ понимаем следовое пространство, снабженное естественной нормой $\left\|V ; V_{\beta}^{l-1}(\partial \Omega)\right\|=\inf \left\{\left\|v ; V_{\beta}^{l}(\Omega)\right\|: v=V\right.$ на $\left.\bar{\Omega} \backslash \mathbf{P}\right\}$. При $k \in \mathbb{N}$ вложение $V_{\beta+k}^{l+k}(\Omega) \subset V_{\beta}^{l}(\Omega)$ непрерывно, но не компактно. В силу неравенств Харди пространства $H^{1}(\Omega)$ и $V_{0}^{1}(\Omega)$ неразличимы, включение $V_{0}^{l}(\Omega) \subset H^{l}(\Omega)$ выполняется при любом $l \in \mathbb{N}_{0}$, а пространство $V_{0}^{2}(\Omega)$ совпадает с подпространством

$$
\left\{v \in H^{2}(\Omega): v\left(P^{1}\right)=\cdots=v\left(P^{J}\right)=0\right\} .
$$

Нетрудно убедиться в том, что оператор задачи (2.6)

$$
\mathscr{O}_{\beta}^{l}=\left\{L^{0}, N^{0}\right\}: V_{\beta}^{l+1}(\Omega)^{n} \rightarrow V_{\beta}^{l-1}(\Omega)^{n} \times V_{\beta}^{l-1 / 2}(\partial \Omega)^{n}
$$


непрерывен при любых $l \in \mathbb{N}$ и $\beta \in \mathbb{R}$. Другие его характеристики и асимптотические свойства решений приведены в следующем утверждении, конкретизирующем общие результаты теории краевых задач в областях с коническими точками на границе (см. ключевые работы [15, 16], а также [12,17] и гл. 4, 6 из [8]). Через ж обозначаем наибольшую степень компонент полиномов в линеале (2.4).

ПРЕДЛОЖЕНИЕ 3.1. Пусть $l \in \mathbb{N} u|\gamma-l+\varkappa+1|<1 / 2$.

1) Оператор $\mathscr{O}_{\gamma}^{l}\left(\right.$ оператор $\left.\mathscr{O}_{2 l-\gamma}^{l}\right)$ является бредгольмовым мономорфизмом (эпиморфизмом), причем

$$
\operatorname{dim} \operatorname{coker} \mathscr{O}_{\gamma}^{l}=\operatorname{dim} \operatorname{ker} \mathscr{O}_{2 l-\gamma}^{l}=J d:=J n(1+\varkappa) .
$$

2) Если $u^{0} \in V_{2 l-\gamma}^{l+1}(\Omega)^{n}$ - решение задачи (2.6) с правыми частями

$$
f^{0} \in V_{\gamma}^{l-1}(\Omega)^{n}, \quad g^{0} \in V_{\gamma}^{l-1 / 2}(\partial \Omega)^{n},
$$

то справедливо асимптотическое представление

$$
u^{0}(x)=\tilde{u}^{0}(x)+\sum_{j=1}^{J} \chi_{j}(x) \sum_{i=1}^{d}\left\{\mathbf{a}_{i}^{j} q^{j, i}(x)+\mathbf{b}_{i}^{j} s^{j, i}(x)\right\},
$$

в котором $\chi_{j} \in C^{\infty}(\bar{\Omega})$ - срезающие функиии с взаимно непересекаюшимися носителями, равные единище в окрестности «своей» точки $P^{j}$,

$$
\tilde{u}^{0} \in V_{\gamma}^{l+1}(\Omega)^{n}, \quad \mathbf{a}^{j}=\left(\mathbf{a}_{1}^{j}, \ldots, \mathbf{a}_{d}^{j}\right)^{\top}, \mathbf{b}^{j}=\left(\mathbf{b}_{1}^{j}, \ldots, \mathbf{b}_{d}^{j}\right)^{\top} \in \mathbb{R}^{d},
$$

$q^{j, i}$ - векторные полиномы степени $\mu_{i}$, вообще говоря, неоднородные (количество полиномов степени $\mu \leqslant \varkappa$ равно $n(1+\mu)), s^{j, i}-$ сингулярные составляющ,

$$
s^{j, i}(x)=\sum_{p=0}^{\varkappa+1+\mu_{i}} r_{j}^{-1-\mu_{i}+p} S_{(p)}^{j, i}\left(\log r_{j}, \varphi^{j}\right)
$$

где $S_{(p)}^{j, i}(l, \cdot)$ - полином переменной l степени не выше $p$ с коэфбициентами - гладкими функииями угловых переменных $\varphi^{j}=r_{j}^{-1}\left(x-P^{j}\right) \in \mathbb{S}^{2}$. Только коэфбициенты $\mathbf{a}_{i}^{j} u \mathbf{b}_{i}^{j}$, но не выражсения $q^{j, i} u s^{j, i}$, зависят от $f^{0}, g^{0} u u^{0}$. Справедливо неравенство

$$
\begin{aligned}
& \left\|\tilde{u}^{0} ; V_{\gamma}^{l+1}(\Omega)\right\|^{2}+\sum_{j=1}^{J}\left(\left|\mathbf{a}^{j}\right|^{2}+\left|\mathbf{b}^{j}\right|^{2}\right) \\
& \quad \leqslant c\left\{\left\|f^{0} ; V_{\gamma}^{l-1}(\Omega)\right\|^{2}+\left\|g^{0} ; V_{\gamma}^{l-1 / 2}(\partial \Omega)\right\|^{2}+\left\|u^{0} ; V_{2 l-\gamma}^{l+1}(\Omega)\right\|^{2}\right\} .
\end{aligned}
$$

Выполняются включения $L^{0} u^{0} \in V_{\gamma}^{l-1}(\Omega)^{n} \subset V_{\gamma-l+1}^{0}(\Omega)^{n}$ u $N^{0} u^{0} \in V_{\gamma}^{l-1 / 2}(\partial \Omega)^{n}$ $\subset V_{\gamma-l+1 / 2}^{0}(\partial \Omega)^{n}$, причем соответствующие нормы не превосходят левой части неравенства (3.9), умноженной на постоянную.

3) При фиксированном наборе полиномов $\left\{q^{j, i}\right\}$ угловые части $S_{(p)}^{j, i}$ в $(3.8)$ можно выбрать так, чтобы для любых вектор-функиий $u^{0}$ u $v^{0}$, допускающих 
разложение (3.6) со столбиами коэффициентов $\mathbf{a}_{u}^{j}, \mathbf{b}_{u}^{j} u \mathbf{a}_{v}^{j}, \mathbf{b}_{v}^{j}$ соответственно, выполнялась обобщенная формула Грина

$$
\left(L^{0} u^{0}, v^{0}\right)_{\Omega}+\left(N^{0} u^{0}, v^{0}\right)_{\partial \Omega}-\left(u^{0}, L^{0} v^{0}\right)_{\Omega}-\left(u^{0}, N^{0} u^{0}\right)_{\partial \Omega}=\sum_{j=1}^{J}\left(\left\langle\mathbf{a}_{u}^{j}, \mathbf{b}_{v}^{j}\right\rangle-\left\langle\mathbf{b}_{u}^{j}, \mathbf{a}_{v}^{j}\right\rangle\right),
$$

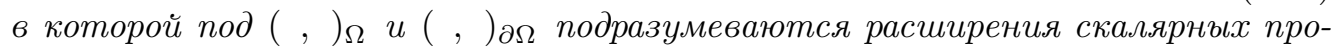
изведений в $L^{2}(\Omega)^{n}$ и $L^{2}(\partial \Omega)^{n}$ до двойственности между элементами пар $V_{\sigma-l+1}^{0}(\Omega)^{n}, V_{(2 l-\sigma)-(l+1)}^{0}(\Omega)^{n} u V_{\sigma-l+1 / 2}^{0}(\partial \Omega)^{n}, V_{(2 l-\sigma)-(l+1 / 2)}^{0}(\partial \Omega)^{n} ;$ здесъ $\sigma=\gamma$ или $\sigma=2 l-\gamma$.

ЗАмЕЧАНИЕ 3.1. Число $n(1+\varkappa)$, фигурирующее в $(3.4)$, указывает количество линейно независимых векторных полиномов степени не выше $\varkappa$, удовлетворяющих однородной модельной задаче в полупространстве $\mathbb{R}_{j+}^{3}=\{x$ : $\left.\nu\left(P^{j}\right)^{\top}\left(x-P^{j}\right)<0\right\}$

$$
\begin{aligned}
D^{0}\left(-\nabla_{x}\right)^{\top} A^{0}\left(P^{j}\right) D^{0}\left(\nabla_{x}\right) v(x) & =0, & & x \in \mathbb{R}_{j+}^{3}, \\
D^{0}\left(\nu\left(P^{j}\right)\right)^{\top} A^{0}\left(P^{j}\right) D^{0}\left(\nabla_{x}\right) v(x) & =0, & & x \in \partial \mathbb{R}_{j+}^{3} \backslash\left\{P^{j}\right\} .
\end{aligned}
$$

Предложение 3.1 нуждается в пояснениях.

Благодаря алгебраической комплектности (2.2) матрицы $D^{0}$ задача (2.6) допускает вариационную постановку (см. [13]), причем ее решение определено с точностью до слагаемого из $\mathscr{P}$, а условия разрешимости имеют вид

$$
\left(f^{0}, p\right)_{\Omega}+\left(g^{0}, p\right)_{\partial \Omega}=0 \quad \forall p \in \mathscr{P} .
$$

При учете сказанного о пространствах $V_{\beta}^{l+1}(\Omega)$ перед формулой (3.2) у оператора $\mathscr{O}_{l}^{l}$ ядро и коядро также определены соотношениями (2.4) и (3.12) соответственно; подчеркнем, что, согласно $[15,18]$, отображение $(3.3)$ теряет фредгольмовость лишь при полуцелых показателях $\beta \in \mathbb{Z}+1 / 2$. Поскольку только тривиальный элемент линеала $\mathscr{P}$ попадает в пространство $V_{\gamma}^{l+1}(\Omega)^{n}$ при $\gamma-l+\varkappa<-1 / 2$ (ср. с определением (3.1)), оператор $\mathscr{O}_{\gamma}^{l}$ становится мономорфизмом, а размерность его коядра вычисляется с помощью теоремы о приращении индекса при переходе через запретные значения $\beta \in \mathbb{Z}+1 / 2$ (см., например, теорему 4.3.3 в [8] и замечание 3.1). Операторы $\mathscr{O}_{\gamma}^{l}$ и $\mathscr{O}_{2 l-\gamma}^{l}$ оказываются сопряженными относительно формулы Грина ${ }^{1)}(2.15)_{1}$, перенесенной на весовые классы по замыканию, и поэтому оператор $\mathscr{O}_{2 l-\gamma}^{l}$ обслуживается результатами из [16] (см. также $[8, \S 4.1])$. Асимптотическое разложение $(3.6)$, оценка (3.9) и форма (3.8) сингулярных решений вытекают из [15], а уточнение структур (3.8) и вывод подобных (3.10) обобщенных формул Грина приведены в $[7,17]$.

ЗАмечание 3.2 . Если $\varkappa=0$ и $\mathscr{P}=\mathbb{R}^{n}$ (см. пример 2.1(1)), то можно взять $l=1, \gamma=0$ и, следовательно, пространство $V_{\gamma}^{l+1}(\Omega)=V_{0}^{2}(\Omega)$ совпадает с подпространством (3.2). Решения $u^{0} \in V_{2 l-\gamma}^{l+1}(\Omega)^{n}=V_{2}^{2}(\Omega)^{n}$ также допускают ясную интерпретацию - к классическому решению $\hat{u}^{0} \in H^{2}(\Omega)^{n}$ добавляется

1) Нижний индекс указывает номер строки в соответствующей формуле. 
линейная комбинация обобщенных матриц Грина с особенностями в точках $P^{j}$ (ср. с [19] и см. замечание 3.3):

$$
u^{0}(x)=\hat{u}^{0}(x)+\sum_{j=1}^{J} \mathfrak{G}^{j}(x) \mathbf{b}^{j} .
$$

Для того чтобы написать аналогичное (3.13) представление в общем случае, заменим систему дифференциальных уравнений (2.6) 1 интегро-дифференциальной системой

$$
\begin{aligned}
L^{0}\left(x, \nabla_{x}\right) v^{0}(x)+\zeta(x) \mathbf{p}(x) \Pi\left(v^{0}\right) & =f^{0}(x), & & x \in \Omega, \\
N^{0}\left(x, \nabla_{x}\right) v^{0}(x) & =g^{0}(x), & & x \in \partial \Omega .
\end{aligned}
$$

Здесь $\Pi: L_{2}(\Omega)^{n} \rightarrow \mathbb{R}^{d_{0}}-$ проекция,

$$
\Pi(v)=\left\{\int_{\Omega} \zeta(x)^{2} \mathbf{p}(x)^{\top} \mathbf{p}(x) d x\right\}^{-1} \int_{\Omega} \mathbf{p}(x)^{\top} v(x) d x,
$$

$\mathbf{p}=\left(\mathbf{p}^{1}, \ldots, \mathbf{p}^{d_{0}}\right)$ - строка векторных полиномов, составляющих базис в линеале $\mathscr{P}$, а $\zeta$ - неотрицательная функция из $C_{0}^{\infty}(\Omega)$, равная единице на непустой внутренней подобласти $\Omega^{\prime} \subset \Omega$. Матрица Грама $\Pi_{\Omega}$ из фигурных скобок в $(3.15)$ положительно определена. Следующее утверждение очевидно.

ЛЕмма 3.1. При любых правых частях (2.7) задача (3.14) имеет единственное решение $v^{0} \in H^{2}(\Omega)^{n}$. Если выполнено условие ортогональности (3.12), то решение $v^{0}$ совпадает с решением $u^{0} \in H^{2}(\Omega)^{n}$ задачи $(2.6)$, подчиненным соотношению $\Pi\left(u^{0}\right)=0$.

ЗАМЕчАНИЕ 3.3. По определению столбцы $\mathfrak{G}^{j, k}$ обобщенной матрицы Грина $\mathfrak{G}^{j}$, упомянутой в замечании 3.1 , удовлетворяют задаче $(3.14)$, в которой $f^{0}=0$ и $g^{0}(x)=\delta\left(x-P^{j}\right) e_{k}$, где $e_{k}=\left(\delta_{1, k}, \ldots, \delta_{n, k}\right)^{\top}$ и $\delta-$ функция Дирака. Таким образом, при $v^{0} \in H^{2}(\Omega)^{n}$ и соответственно $\mathbf{b}_{v}^{j}=0$ равенство (3.10) становится формулой Грина в рамках теории распределений: выражение $N^{0} u^{0}$ рассматривается на проколотой поверхности $\partial \Omega \backslash \mathbf{P}$, но происходящий от $\delta$-функции член $v^{0}\left(P^{j}\right)^{\top} \mathbf{b}_{u}^{j}=\left(\mathbf{a}_{v}^{j}\right)^{\top} \mathbf{b}_{u}^{j}$ присутствует в правой части соотношения (3.10). Если оба столбца $\mathbf{b}_{u}^{j}$ и $\mathbf{b}_{v}^{j}$ ненулевые, упомянутая интерпретация формулы Грина затруднительна, а ее проверка осуществляется предельным переходом при $\delta \rightarrow+0$ в формуле Грина $(2.15)_{1}$ на области $\Omega_{\delta}=\left\{x \in \Omega: \operatorname{dist}\left(x, P^{j}\right)>\delta\right\}(\operatorname{cp.~c~[16]~}$ и $[8, \S \S 3.2,3.4])$, причем в $[7,17]$ установлено, что при надлежащем выборе угловых частей $S_{(1)}^{j, i}, \ldots, S_{\left(\varkappa+2+\mu_{i}\right)}^{j, i}$ пределы возникающих интегралов по малым множествам $\left\{x \in \Omega: \operatorname{dist}\left(x, P^{j}\right)=\delta\right\}$ совпадают с членами суммы по $j$ в $(3.10)$.

Через $\mathfrak{G}^{j, i}$ обозначим решение однородной системы уравнений $(3.14)_{1}$, удовлетворяющее однородным краевым условиям (3.14) 2 на $\partial \Omega \backslash P^{j}$ и имеющее сингулярности (3.8) вблизи точки $P^{j}$. Это решение следует искать в виде

$$
\mathfrak{G}^{j, i}(x)=\chi_{j}(x) s^{j, i}(x)+\widehat{\mathfrak{G}}^{j, i}(x), \quad \widehat{\mathfrak{G}}^{j, i} \in H^{\varkappa+2}(\Omega)^{n} .
$$

Действительно, лемма 3.1 и последующее поднятие гладкости решения доставляют остатки $\widehat{\mathfrak{G}}^{j, i}$ с нужными свойствами, поскольку, согласно предложению $3.1(2)$, где $l=\varkappa+1$ и $\gamma=0$, верны включения $L^{0} \chi_{j} s^{j, i} \in V_{\gamma}^{l-1}(\Omega)^{n} \subset H^{\varkappa}(\Omega)^{n}$ и 
$L^{0} \chi_{j} s^{j, i} \in V_{\gamma}^{l-1 / 2}(\partial \Omega)^{n} \subset H^{\varkappa+1 / 2}(\partial \Omega)^{n}$ (см. текст перед формулой (3.2)). Подчеркнем, что интегрирование в (3.15) и добавка в (3.14) удалены от границы $\partial \Omega$ и поэтому никак не затрагивают сингулярности в точках $P^{j}$.

\section{§4. Асимптотические условия сопряжения в точках $P^{j}$}

Введем пространство

$$
\mathfrak{H}=\mathfrak{D} \times \mathscr{\mathscr { H }}^{\mathbf{t}}\left(\Upsilon^{1}\right) \times \cdots \times \mathscr{\mathscr { H }}^{\mathbf{t}}\left(\Upsilon^{J}\right)
$$

функций-столбцов $\mathbf{u}=\left(u^{0}, u^{1}, \ldots, u^{J}\right)^{\top}$ и сделаем его гильбертовым, определив норму по формуле

$$
\|\mathbf{u} ; \mathfrak{H}\|=\left\{\left\|\tilde{u}^{0} ; V_{\gamma}^{2}(\Omega)\right\|^{2}+\sum_{j=1}^{J}\left(\left|\mathbf{a}^{j}\right|^{2}+\left|\mathbf{b}^{j}\right|^{2}+\left\|u^{j} ; \stackrel{\mathscr{H}}{\mathbf{t}}^{\mathbf{t}}\left(\Upsilon^{j}\right)\right\|^{2}\right)\right\}^{1 / 2} .
$$

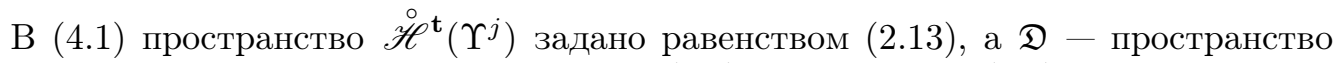
вектор-функций, представимых в виде (3.6) с атрибутами (3.7), причем показатели гладкости и веса фиксируем так:

$$
l=1, \quad \gamma=-\varkappa .
$$

Ясно, что модификация срезающих функций $\chi_{j}$ в (3.6) не сказывается на пространствах $\mathfrak{H}$ и $\mathfrak{D}$ ни алгебраически, ни топологически. Введем на $\mathfrak{H}$ проекции $\pi_{ \pm}^{j}$ и $\pi^{j}$ равенствами $\pi_{+}^{j} \mathbf{u}=\mathbf{a}^{j}, \pi_{-}^{j} \mathbf{u}=\mathbf{b}^{j}$ и $\pi^{j} \mathbf{u}=u^{j}-$ условия сопряжения в точках реализуются как связи, налагаемые на $\pi_{ \pm}^{j} \mathbf{u} \in \mathbb{R}^{d}$ и $D^{j}\left(\partial_{z^{j}}\right) \pi^{j} \mathbf{u}(0)$, $N^{j}\left(\partial_{z^{j}}\right) \pi^{j} \mathbf{u}(0) \in \mathbb{R}^{d_{0}}$.

Линейная оболочка полиномиальных столбцов $q^{j, 1}, \ldots, q^{j, d}$ из предложения $3.1(2)$ включает в себя подпространство (2.4) (потому что элемент $p \in \mathscr{P}$ удовлетворяет однородной задаче (2.6)). Согласованным изменением базисов $\left\{q^{j, i}\right\}$ и $\left\{s^{j, i}\right\}$ гладких и сингулярных составляющих разложения (3.6) добиваемся при сохранении формулы (3.10) совпадения строк $\left(q^{j, 1}, \ldots, q^{j, d_{0}}\right)$ и

$$
\left(p_{(j)}^{1,0}, \ldots, p_{(j)}^{1, t^{1}-1}, \ldots, p_{(j)}^{T, 0}, \ldots, p_{(j)}^{T, t^{T}-1}\right),
$$

где $\left\{p_{(j)}^{k, i}\right\}$ - базис в $\mathscr{P}$, упомянутый в $\S 2$, а порядок расположения элементов в (4.4) аналогичен (2.11). За столбцом $a^{j} \in \mathbb{R}^{d_{0}}$, увеличенным до высоты $d$ путем добавления нулей снизу, сохраняем обозначение $a^{j}$, а столбец $\mathbf{a}^{j} \in \mathbb{R}^{d}$, из которого удалены нижние $d-d_{0}$ элементов, обозначаем через $\mathbf{a}_{\#}^{j}$. Далее нам понадобятся два ортогональных проектора $\mathfrak{P}^{j}$ в $\mathbb{R}^{d}$ и $\mathfrak{p}^{j}$ в $\mathbb{R}^{d_{0}}$, а также два симметрических оператора $\mathfrak{S}^{j}$ и $\mathfrak{s}^{j}$ на подпространствах $\mathfrak{P}^{j} \mathbb{R}^{d}$ и $\mathfrak{p}^{j} \mathbb{R}^{d_{0}}$ соответственно.

В качестве искомых условий сопряжения в точках $P^{1}, \ldots, P^{J}$ возьмем

$$
\begin{gathered}
\mathfrak{P}^{j} \pi_{+}^{j} \mathbf{u}-\mathfrak{S}^{j} \mathfrak{P}^{j} \pi_{-}^{j} \mathbf{u}-\mathfrak{P}^{j} \mathfrak{s}^{j} \mathfrak{p}^{j} D^{j}\left(\pi^{j} \mathbf{u}\right)(0)=0, \\
\mathfrak{s}^{j} \mathfrak{p}^{j}\left(\pi_{-}^{j} \mathbf{u}\right)_{\#}+\mathfrak{p}^{j} N^{j}\left(\pi^{j} \mathbf{u}\right)(0)=0, \\
\left(\mathbb{I}_{d}-\mathfrak{P}^{j}\right) \pi_{-}^{j} \mathbf{u}=0, \\
\left(\mathbb{I}_{d_{0}}-\mathfrak{p}^{j}\right) D^{j}\left(\pi^{j} \mathbf{u}\right)(0)=0, \quad j=1, \ldots, J ;
\end{gathered}
$$


здесь $D^{j}$ и $N^{j}$ - граничные дифференциальные операторы, фигурирующие в $(2.15)_{2}$, а $\mathbb{I}_{d}$ - тождественное отображение в пространстве $\mathbb{R}^{d}$. Асимптотическая и механическая подоплека условий (4.5) будет разъяснена в $\S 7$, а сейчас займемся исследованием задачи (2.6), (2.8) и (2.10) с асимптотическими условиями сопряжения (4.5), которую кратко обозначим $(\star)$ (ср. с $(1.1))$.

ЛЕмма 4.1. Для любых $\mathbf{u}, \mathbf{v} \in \mathfrak{H}$ справедлива обобщенная формула Грина

$$
\mathfrak{q}(\mathbf{u}, \mathbf{v})=\mathfrak{q}(\mathbf{v}, \mathbf{u})
$$

где при использовании обозначений из (3.10), (3.6) и (2.14)

$$
\begin{aligned}
\mathfrak{q}(\mathbf{u}, \mathbf{v})= & \left(L^{0} u^{0}, v^{0}\right)_{\Omega}+\left(N^{0} u^{0}, v^{0}\right)_{\partial \Omega} \\
& +\sum_{j=1}^{J}\left\{\left(L^{j} u^{j}, v^{j}\right)_{\Upsilon^{j}}-\left\langle\left(\mathbb{I}_{d}-\mathfrak{P}^{j}\right) \mathbf{b}_{u}^{j}, \mathbf{a}_{v}^{j}+\mathfrak{s}^{j} \mathfrak{p}^{j} D^{j} v^{j}(0)\right\rangle\right. \\
& \quad-\left\langle\left(\mathbb{I}_{d_{0}}-\mathfrak{p}^{j}\right) D^{j} u^{j}(0), N^{j} v^{j}(0)\right\rangle+\left\langle\mathfrak{s}^{j} \mathfrak{p}^{j}\left(\mathbf{b}_{u}^{j}\right)_{\#}+\mathfrak{p}^{j} N^{j} u^{j}(0), D^{j} v^{j}(0)\right\rangle \\
& \left.-\left\langle\mathfrak{P}^{j} \mathbf{a}_{u}^{j}-\mathfrak{S}^{j} \mathfrak{P}^{j} \mathbf{b}_{u}^{j}-\mathfrak{P}^{j} \mathfrak{s}^{j} \mathfrak{p}^{j} D^{j} u^{j}(0), \mathbf{b}_{v}^{j}\right\rangle\right\} . \quad(4.7)
\end{aligned}
$$

ДоказАтельство. Сложим формулы Грина $(3.10)$ и $(2.15)_{2}, j=1, \ldots, J$, после чего сделаем следующее преобразование, использующее симметричность операторов:

$$
\begin{gathered}
\left\langle\mathbf{a}_{u}^{j}, \mathfrak{P}^{j} \mathbf{b}_{v}^{j}\right\rangle-\left\langle\mathfrak{P}^{j} \mathbf{b}_{u}^{j}, \mathbf{a}_{v}^{j}\right\rangle+\left\langle\mathfrak{p}^{j} D^{j} u^{j}(0), N^{j} v^{j}(0)\right\rangle-\left\langle N^{j} u^{j}(0), \mathfrak{p}^{j} D^{j} v^{j}(0)\right\rangle \\
=\left\langle\mathfrak{P}^{j} \mathbf{a}_{u}^{j}-\mathfrak{S}^{j} \mathfrak{P}^{j} \mathbf{b}_{u}^{j}, \mathbf{b}_{v}^{j}\right\rangle-\left\langle\mathbf{b}_{u}^{j}, \mathfrak{P}^{j} \mathbf{a}_{v}^{j}-\mathfrak{S}^{j} \mathfrak{P}^{j} \mathbf{b}_{v}^{j}\right\rangle \\
\quad-\left\langle\mathfrak{p}^{j} N^{j} u^{j}(0)+\mathfrak{s}^{j} \mathfrak{p}^{j}\left(\mathbf{b}_{u}^{j}\right)_{\#}, D^{j} v^{j}(0)\right\rangle+\left\langle\mathfrak{s}^{j} \mathfrak{p}^{j}\left(\mathbf{b}_{u}^{j}\right)_{\#}, D^{j} v^{j}(0)\right\rangle \\
\quad+\left\langle D^{j} u^{j}(0), \mathfrak{p}^{j} N^{j} v^{j}(0)+\mathfrak{s}^{j} \mathfrak{p}^{j}\left(\mathbf{b}_{v}^{j}\right)_{\#}\right\rangle-\left\langle D^{j} u^{j}(0), \mathfrak{s}^{j} \mathfrak{p}^{j}\left(\mathbf{b}_{v}^{j}\right)_{\#}\right\rangle .
\end{gathered}
$$

Осталось переписать член $\left\langle D^{j} u^{j}(0), \mathfrak{s}^{j} \mathfrak{p}^{j}\left(\mathbf{b}_{v}^{j}\right)_{\#}\right\rangle$ (и аналогичный ему) в виде $\left\langle\mathfrak{P}^{j} \mathfrak{s}^{j} \mathfrak{p}^{j} D^{j} u^{j}(0), \mathbf{b}_{v}^{j}\right\rangle+\left\langle\mathfrak{s}^{j} \mathfrak{p}^{j} D^{j} u^{j}(0),\left(\mathbb{I}_{d}-\mathfrak{P}^{j}\right) \mathbf{b}_{v}^{j}\right\rangle$ и распределить слагаемые по составляющим в (4.7).

Итак, задача $(\star)$ включается в симметричную обобщенную формулу Грина, т. е. оказывается формально самосопряженной. Очередное утверждение о повышении гладкости решения очевидно.

ПРЕДЛОЖЕНИЕ 4.1. Пусть и - решение задачи ( $\star$ ) из пространства (4.1) с индексами (4.3), но справедливы включения (3.5) и

$$
f^{j} \in \mathscr{H}^{s-\mathbf{t}}\left(\Upsilon^{j}\right)=H^{s-t_{1}}\left(\Upsilon^{j}\right) \times \cdots \times H^{s-t_{T}}\left(\Upsilon^{j}\right),
$$

где $l \geqslant 1, s \geqslant \max \mathbf{t}$, а пара $l, \gamma$ подчинена условию из предложсения 3.1. Тогда компонента $u^{0}$ решения $\mathbf{u}$ принадлежит пространству $V_{2 l-\gamma}^{l+1}(\Omega)^{n}$ и допускает представление (3.6) с атрибутами (3.7), а компоненты $u^{j}$ попадают в пространства $\mathscr{H}^{s+\mathbf{t}}\left(\Upsilon^{j}\right)$, причем соответствующие нормы компонент $u^{0} u$ $u^{j}$ не превосходят постоянной, умноженной на сумму норм правых частей (3.5) u (4.8), а также нормы $\|\mathbf{u} ; \mathfrak{H}\|$.

Для задачи $(\star)$ верна альтернатива Фредгольма. 
ПРЕДЛОЖКНИЕ 4.2. Пустъ $\mathfrak{h}-$ подпространство решений $\mathbf{u} \in \mathfrak{H}$ однородной

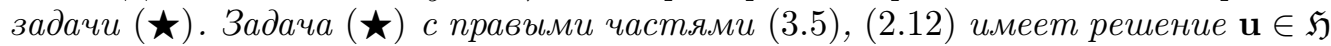
в том и только в том случае, когда

$$
\left(f^{0}, v^{0}\right)_{\Omega}+\left(g^{0}, v^{0}\right)_{\partial \Omega}+\sum_{j=1}^{J}\left(f^{j}, v^{j}\right)_{\Upsilon^{j}}=0 \quad \forall \mathbf{v} \in \mathfrak{h} .
$$

В частности, индекс оператора $\mathfrak{O}_{\star}$ задачи $(\star)$ равен нулю.

ДокАЗАТЕЛЬСтво. Необходимость условий разрешимости (4.9) вытекает из обобщенной формулы Грина (4.6), а достаточность обеспечивается равенством Ind $\mathfrak{O}_{\star}=0$. Проверим его. Оператор $\mathfrak{O}_{\star}$ фредгольмов, так как лишь конечномерным возмущением отличается от оператора $\mathscr{O}=\left(\mathscr{O}_{0}^{2}, \mathscr{O}_{1}, \ldots, \mathscr{O}_{J}\right)^{\top}$ с областью определения $\mathscr{D}(\mathscr{O})$; при этом $\mathscr{O}_{0}^{2}$ взят из $(3.3)$, а $\mathscr{O}_{j}-$ оператор задачи Дирихле для системы (2.8), т. е. $\left(L^{j},\left.D^{j}\right|_{z^{j}=0}\right): \mathscr{\mathscr { H }}^{\mathbf{t}}\left(\Upsilon^{j}\right) \rightarrow \mathscr{H}^{-\mathbf{t}}\left(\Upsilon^{j}\right) \times \mathbb{R}^{d_{0}}$. Cогласно предложению 3.1(1) индекс оператора $\mathscr{O}$ равен $-J d$. Так как факторпространство $\mathfrak{H} / \mathscr{D}(\mathscr{O})$ имеет размерность $J\left(2 d+d_{0}\right)$, а условия $(4.5)$ налагают связи в количестве

$$
J\left\{\operatorname{dim}\left(\mathfrak{P}^{j} \mathbb{R}^{d}\right)+\operatorname{dim}\left(\mathfrak{p}^{j} \mathbb{R}^{d_{0}}\right)+\operatorname{codim}\left(\mathfrak{P}^{j} \mathbb{R}^{d}\right)+\operatorname{codim}\left(\mathfrak{p}^{j} \mathbb{R}^{d_{0}}\right)\right\}=J\left(d+d_{0}\right),
$$

получаем нужное равенство Ind $\mathfrak{O}_{\star}=-J d+J\left(2 d+d_{0}\right)-J\left(d+d_{0}\right)=0$.

СлЕДСТВиЕ 4.1. Если размерность подпространства

$$
\mathscr{P}^{\star}=\left\{p \in \mathscr{P}: \mathfrak{P}^{j} \pi_{+}^{j}(p, 0, \ldots, 0)^{\top}=0, j=1, \ldots, J\right\}
$$

положительна, задача $(\star)$ не может быть однозначно разрешимой.

ДоказАтельство. При $p \in \mathscr{P} \star$ столбец $\mathbf{w}=(p, 0, \ldots, 0)^{\top}$ удовлетворяет всем условиям сопряжения в точках $P^{j}$, поскольку $\pi_{-}^{j} \mathbf{w}=0, \pi^{j} \mathbf{w}=0$, а в (4.5) фигурирует только проекция $\mathfrak{P}^{j} \pi_{+}^{j} \mathbf{w}$, равная нулю в силу (4.10). СледовательHо, $\mathscr{P} \star \subset \mathfrak{h}$.

\section{§5. Вариационная формулировка задачи}

Рассмотрим на пространстве $\mathfrak{H}$ квадратичную форму

$$
\mathfrak{Q}(\mathbf{u})=\frac{1}{2} \mathfrak{q}(\mathbf{u}, \mathbf{u})-\mathfrak{f}(\mathbf{u}),
$$

где форма $\mathfrak{q}(\mathbf{u}, \mathbf{v})$ определена равенством (4.7) и

$$
\mathfrak{f}(\mathbf{u})=\left(f^{0}, u^{0}\right)_{\Omega}+\left(g^{0}, u^{0}\right)_{\partial \Omega}+\sum_{j=1}^{J}\left(f^{j}, u^{j}\right)_{\Upsilon^{j}} .
$$

ПрЕДЛОЖЕНИЕ 5.1. Столбеи, $\mathbf{u} \in \mathfrak{H}$ является стачионарной точкой функиионала (5.1) тогда и только тогда, когда этот столбеи - решение задачи $(\star)$. 
ДокАЗАТЕЛЬСТво. Вычисляя вариацию формы (5.1) и применяя формулу Грина (4.6), обнаруживаем, что

$$
\begin{aligned}
\delta \mathfrak{Q}(\mathbf{u}, \mathbf{v})=\frac{1}{2} \mathfrak{q}(\mathbf{u}, \mathbf{v})+ & \frac{1}{2} \mathfrak{q}(\mathbf{v}, \mathbf{u})-\mathfrak{f}(\mathbf{v})=\mathfrak{q}(\mathbf{u}, \mathbf{v})-\mathfrak{f}(\mathbf{v}) \\
=\left(L^{0} u^{0}-f^{0}, v^{0}\right)_{\Omega}+\left(N^{0} u^{0}-g^{0}, v^{0}\right)_{\partial \Omega} & \\
+\sum_{j=1}^{J}\{ & \left(L^{j} u^{j}-f^{j}, v^{j}\right)_{\Upsilon j}-\left\langle\left(\mathbb{I}_{d}-\mathfrak{P}^{j}\right) \pi_{-}^{j} \mathbf{u}, \pi_{+}^{j} \mathbf{v}+\mathfrak{s}^{j} \mathfrak{p}^{j} D^{j}\left(\pi^{j} \mathbf{v}\right)(0)\right\rangle \\
& -\left\langle\left(\mathbb{I}_{d_{0}}-\mathfrak{p}^{j}\right) D^{j}\left(\pi^{j} \mathbf{u}\right)(0), N^{j}\left(\pi^{j} \mathbf{v}\right)(0)\right\rangle \\
& +\left\langle\mathfrak{s}^{j} \mathfrak{p}^{j}\left(\pi_{-}^{j} \mathbf{u}\right)_{\#}+\mathfrak{p}^{j} N^{j}\left(\pi^{j} \mathbf{u}\right)(0), D^{j}\left(\pi^{j} \mathbf{v}\right)(0)\right\rangle \\
& \left.-\left\langle\mathfrak{P}^{j} \pi_{+}^{j} \mathbf{u}-\mathfrak{S}^{j} \mathfrak{P}^{j} \pi_{-}^{j} \mathbf{u}-\mathfrak{P}^{j} \mathfrak{s}^{j} \mathfrak{p}^{j} D^{j}\left(\pi^{j} \mathbf{u}\right)(0), \pi_{-}^{j} \mathbf{v}\right\rangle\right\} .
\end{aligned}
$$

Итак, действительно вариация (5.2) вырождается на решении задачи ( $\star$ ). Если же она сама равна нулю, то возьмем «пробную функцию» $\mathbf{v}$ с компонентами $v^{0} \in C_{0}^{\infty}(\bar{\Omega} \backslash \mathbf{P})^{n}$ и $v^{j} \in C_{0}^{\infty}\left(\Upsilon^{j}\right)^{T}$. Так как проекции $\pi_{ \pm}^{j} \mathbf{v}$ и $D^{j}\left(\pi^{j} \mathbf{v}\right)(0)$, $N^{j}\left(\pi^{j} \mathbf{v}\right)(0)$ нулевые, равенство $\delta \mathfrak{Q}(\mathbf{u}, \mathbf{v})=0$ ввиду произвольности $v^{0}$ и $v^{j}$ обеспечивает соотношения (2.6) и (2.8). При $\mathbf{v} \in \mathfrak{H}$ упомянутые проекции могут быть любыми столбцами в $\mathbb{R}^{d}$ и $\mathbb{R}^{d_{0}}$, а значит, из того же равенства вытекают условия сопряжения (4.5).

Обозначим через $\mathfrak{H}^{0}$ подпространство, составленное из столбцов, удовлетворяющих соотношениям $(4.5)_{2-4}$, которые не затрагивают проекции $\pi_{+}^{1} \mathbf{u}, \ldots, \pi_{+}^{j} \mathbf{u}$. Следовательно, $\mathscr{P}_{\star}:=\mathscr{P} \times\{0\}^{J} \subset \mathfrak{H}^{0}$ и справедливо разложение

$$
\mathfrak{H}^{0}=\mathscr{P}_{\star}+\mathfrak{H}_{\perp}^{0},
$$

где $\mathfrak{H}_{\perp}^{0}=\left\{\mathbf{v} \in \mathfrak{H}^{0}: \Pi\left(\hat{v}^{0}\right)=0 \in \mathbb{R}^{d_{0}}\right\}, \Pi$ - проекция (3.15), содержащая матрицу Грама $\Pi_{\Omega}$, и $\hat{v}^{0}-$ регулярная часть компоненты $v^{0}$, т. е. при $\mathbf{b}^{j}=\pi_{-}^{j} \mathbf{v}$

$$
\hat{v}^{0}=v^{0}-\sum_{j=1}^{J} \sum_{i=1}^{d} \mathbf{b}_{i}^{j} \mathfrak{G}^{j, i} .
$$

Кроме того, введем матрицы $\mathscr{G}^{j, m}$ и $\mathbf{G}^{j, m}$ размера $d \times d$

$$
\begin{gathered}
\mathscr{G}^{j, m}=\left[\left\langle\Pi_{\Omega} \Pi\left(\mathfrak{G}^{j, i}\right), \Pi\left(\mathfrak{G}^{j, i}\right)\right\rangle\right]_{i, k=1}^{d}, \\
\mathbf{G}^{j, m}=\left(\mathbf{G}_{1}^{j, i}, \ldots, \mathbf{G}_{d}^{j, m}\right)^{\top}, \quad \mathbf{G}_{i}^{j, m}=\pi_{+}^{m}\left(\mathfrak{G}^{j, i}, 0, \ldots, 0\right)^{\top} \in \mathbb{R}^{d_{0}} .
\end{gathered}
$$

Столбец $\mathbf{G}_{i}^{j, m}$ совпадает со столбцом $\mathbf{a}^{m}$ коэффициентов при полиномах $q^{m, k}$ в разложении (3.5) сингулярного решения $\mathfrak{G}^{j, i}$, и, следовательно, формула Грина (3.10), примененная к $\mathfrak{G}^{j, i}$ и $\mathfrak{G}^{m, k}$, устанавливает равенство $\mathbf{G}_{i, k}^{j, m}=\mathbf{G}_{k, i}^{m, j}$. Иными словами, матрица $\mathbf{G}$, имеющая размер $J d \times J d$ и составленная из блоков $\mathbf{G}^{j, m}$, оказывается симметрической. По определению $(5.5)_{1}$ тем же свойством обладает и аналогичная матрица $\mathscr{G}$; к тому же она неотрицательно определена. Далее под $\mathfrak{S}$ и $\mathfrak{P}$ подразумеваются блочно-диагональные матрицы $\operatorname{diag}\left\{\mathfrak{S}^{1}, \ldots, \mathfrak{S}^{J}\right\}$ и $\operatorname{diag}\left\{\mathfrak{P}^{1}, \ldots, \mathfrak{P}^{J}\right\}$. 
Теорема 5.1. Пусть $\mathbf{u} \in \mathfrak{H}-$ решение задачи $(\star)$ с правыми частями (3.5) u (2.12). Если оператор

$$
\mathfrak{P}(\mathfrak{S}+\mathscr{G}-\mathbf{G}) \mathfrak{P}: \prod_{j=1}^{J} \mathfrak{P}^{j} \mathbb{R}^{d} \rightarrow \prod_{j=1}^{J} \mathfrak{P}^{j} \mathbb{R}^{d}
$$

положительно определен, то в точке $\mathbf{u}$ функционал (5.1) достигает строгого минимума на множестве $\mathbf{u}+\mathfrak{H}_{\perp}^{0}=\left\{\mathbf{u}+\mathbf{v}: \mathbf{v} \in \mathfrak{H}_{\perp}^{0}\right\}$.

ДокАЗАТЕльство. Для решения $\mathbf{u} \in \mathfrak{H}$ вариация (5.2) вырождается, и поэтому

$$
\frac{1}{2} \mathfrak{q}(\mathbf{u}+\mathbf{v}, \mathbf{u}+\mathbf{v})-\mathfrak{f}(\mathbf{u}+\mathbf{v})-\left(\frac{1}{2} \mathfrak{q}(\mathbf{u}, \mathbf{u})-\mathfrak{f}(\mathbf{u})\right)=\frac{1}{2} \mathfrak{q}(\mathbf{v}, \mathbf{v}) .
$$

Используя равенства (5.4), (4.5) 2-4 и интегрируя по частям (cp. (3.10), а также $(2.5)$ и аналогичные формулы на отрезках $\left.\Upsilon^{j}\right)$, получаем, что

$$
\begin{aligned}
\mathfrak{q}(\mathbf{v}, \mathbf{v})= & \left(L^{0} v^{0}, v^{0}\right)_{\Omega}+\left(N^{0} v^{0}, v^{0}\right)_{\partial \Omega} \\
& +\sum_{j=1}^{J}\left\{\left(L^{j} v^{j}, v^{j}\right)_{\Upsilon j}+\left\langle\mathfrak{S}^{j} \mathfrak{P}^{j} \mathbf{b}^{j}-\mathfrak{P}^{j} \mathbf{a}^{j}+\mathfrak{P}^{j} \mathfrak{s}^{j} \mathfrak{p}^{j} D^{j} v^{j}(0), \mathbf{b}^{j}\right\rangle\right\} \\
= & \left(L^{0} \hat{v}^{0}, \hat{v}^{0}\right)_{\Omega}+\left(N^{0} \hat{v}^{0}, \hat{v}^{0}\right)_{\partial \Omega}+\left(\zeta \mathbf{p} \sum_{\cdots} \mathbf{b}_{i}^{j} \Pi\left(\mathfrak{G}^{j, i}\right), \sum_{\cdots} \mathbf{b}_{i}^{j} \mathfrak{G}^{j, i}\right)_{\Omega} \\
& +\left(L^{0} \hat{v}^{0}, \sum_{\cdots} \mathbf{b}_{i}^{j} \mathfrak{G}^{j, i}\right)_{\Omega}+\left(N^{0} \hat{v}^{0}, \sum_{\ldots} \mathbf{b}_{i}^{j} \mathfrak{G}^{j, i}\right)_{\partial \Omega} \\
& +\sum_{j=1}^{J}\left\{\left(L^{j} v^{j}, v^{j}\right)_{\Upsilon j}+\left\langle\mathfrak{S}^{j} \mathfrak{P}^{j} \mathbf{b}^{j}-\mathfrak{P}^{j} \mathbf{a}^{j}+\mathfrak{P}^{j} \mathfrak{s}^{j} \mathfrak{p}^{j} D^{j} v^{j}(0), \mathbf{b}^{j}\right\rangle\right\} \\
= & \left(A^{0} D^{0} \hat{v}^{0}, D^{0} \hat{v}^{0}\right)_{\Omega}+\sum_{j=1}^{J}\left(\left\langle\widehat{\mathbf{a}}^{j}, \mathbf{b}^{j}\right\rangle+\sum_{m=1}^{J}\left\langle\mathscr{G}^{j, m} \mathbf{b}^{j}, \mathbf{b}^{m}\right\rangle\right) \\
& +\sum_{j=1}^{J}\left\{\left(A^{j} \mathscr{D} v^{j}, \mathscr{D} v^{j}\right)_{\Upsilon j}+\left\langle\left(N^{j} v^{j}(0), D^{j} v^{j}(0)\right\rangle\right.\right. \\
& \left.+\left\langle\mathfrak{S}^{j} \mathfrak{P}^{j} \mathbf{b}^{j}-\mathfrak{P}^{j} \mathbf{a}^{j}+\mathfrak{P}^{j} \mathfrak{s}^{j} \mathfrak{p}^{j} D^{j} v^{j}(0), \mathfrak{P}^{j} \mathbf{b}^{j}\right\rangle\right\} \\
= & \left(A^{0} D^{0} \hat{v}^{0}, D^{0} \hat{v}^{0}\right)_{\Omega}+\sum_{j=1}^{J}\left\{\left(A^{j} \mathscr{D} v^{j}, \mathscr{D} v^{j}\right)_{\Upsilon^{j}}\right. \\
& \left.+\sum_{m=1}^{J}\left\langle\left\{\delta_{j, m} \mathfrak{S}^{j}+\mathscr{G}^{j, m}-\mathbf{G}^{j, m}\right\} \mathfrak{P}^{j} \mathbf{b}^{j}, \mathfrak{P}^{m} \mathbf{b}^{m}\right\rangle\right\} .
\end{aligned}
$$

При этом $\widehat{\mathbf{a}}^{j}=\pi_{+}^{j}\left(\hat{v}^{0}, 0, \ldots, 0\right)^{\top}$, а в конце учтено, что в согласии с $(4.5)_{4}$

$$
\begin{aligned}
\pi_{+}^{j} \mathbf{v} & =\mathbf{a}^{j}=\widehat{\mathbf{a}}^{j}+\sum_{m=1}^{J} \mathbf{G}^{m, j} \mathbf{b}^{m}, \\
\left\langle N^{j} v^{j}(0), D^{j} v^{j}(0)\right\rangle & =\left\langle D^{j} v^{j}(0), \mathfrak{p}^{j} N^{j} v^{j}(0)\right\rangle \\
& =-\left\langle D^{j} v^{j}(0), \mathfrak{s}^{j} \mathfrak{p}^{j} \mathbf{b}_{\#}^{j}\right\rangle=-\left\langle\mathfrak{s}^{j} \mathfrak{p}^{j} D^{j} v^{j}(0), \mathfrak{p}^{j} \mathbf{b}^{j}\right\rangle .
\end{aligned}
$$


При положительно определенном операторе (5.6) выражение (5.7) не может быть отрицательным; если оно обращается в нуль, то $\mathfrak{P}^{j} \mathbf{b}^{j}=0, D^{0} \hat{v}^{0}=0$ и $D^{j} v^{j}(0)=0$, а значит, в силу $(4.5)_{3},(2.3),(2.10)$ и равенства $\Pi\left(\hat{v}^{0}\right)=0$ имеем $\mathbf{b}^{j}=0, \hat{v}^{0}=0$ и $v^{j}(0)=0$, т. е. $\mathbf{v}=0$.

ЗАмЕчАниЕ 5.1. Если изменить задачу (2.6) на теле $\Omega$, сделав ее однозначно разрешимой в $H^{2}(\Omega)^{n}$, например, за счет постановки условия Дирихле на компоненте связности $Г$ границы $\partial \Omega$ (при этом $\Gamma \cap \mathbf{P}=\varnothing)$, то проекция $(3.15)$ выродится и упомянутый в теореме 5.1 строгий минимум будет достигаться на всем пространстве $\mathfrak{H}^{0}$.

\section{§6. Самосопряженные расширения}

В этом параграфе краевое условие Неймана $(2.6)_{2}$ считаем однородным. Пусть $\mathscr{A}_{0}^{0}$ - неограниченный оператор в $L_{2}(\Omega)^{n}$ с дифференциальным выражением (2.1) и областью определения $\mathscr{D}\left(\mathscr{A}_{0}^{0}\right)=\left\{u^{0} \in C_{0}^{\infty}(\bar{\Omega} \backslash \mathbf{P})^{n}: N^{0} u^{0}=0\right.$ на $\partial \Omega\}$. Следующее утверждение известно (см. [20] и др.), а его формулировку, апеллирующую к весовым классам, можно найти в [21].

Лемма 6.1. Замыкание $\mathscr{A}_{0}=\overline{\mathscr{A}}_{0}^{0}$ и сопряженный оператор $\mathscr{A}_{0}^{*}$ заданы тем же дифберенииальным выражением, что и $\mathscr{A}_{0}^{0}$, но обладают такими областями определения:

$$
\begin{aligned}
\mathscr{D}\left(\mathscr{A}_{0}\right)=\left\{u^{0} \in V_{0}^{2}(\Omega)^{n}:\right. & \left.N^{0} u^{0}=0 \text { на } \partial \Omega\right\}, \\
\mathscr{D}\left(\mathscr{A}_{0}^{*}\right)=\left\{u^{0} \in V_{2}^{2}(\Omega)^{n}:\right. & N^{0} u^{0}=0 \text { на } \partial \Omega, \\
& \left.u^{0} \text { имеет вид }(3.13) \text { с } \hat{u}^{0} \in V_{1}^{2}(\Omega)^{n}, \mathbf{b}^{j} \in \mathbb{R}^{n}\right\} .
\end{aligned}
$$

Матрица (2.9) содержит дифференциальные операторы второго порядка в том случае, когда $\mathscr{P}=\mathbb{R}^{n}$, а значит, $T=n$ и $\varkappa=0$. Нетрудно проверить, что оператор $\mathscr{A}^{j}$ с дифференциальным выражением $L^{j}\left(z, \partial_{z}\right)=-\partial_{z} A^{j}(z) \partial_{z}$ и областью определения

$$
\mathscr{D}\left(\mathscr{A}_{j}\right)=\left\{u^{j} \in H^{2}\left(\Upsilon^{j}\right)^{n}: u^{j}(0)=\partial_{z} u^{j}(0)=u^{j}\left(l_{j}\right)=0\right\} \subset L_{2}\left(\Upsilon^{j}\right)^{n}
$$

является замкнутым, а для его сопряженного $\mathscr{A}_{j}^{*}$ имеет место равенство $\mathscr{D}\left(\mathscr{A}_{j}^{*}\right)$ $=\mathscr{\mathscr { H }}^{1}\left(\Upsilon^{j}\right)$ (см. замечание 3.1 и формулу $\left.(2.13)\right)$. Таким образом, область определения оператора $\mathscr{A}^{*}=\left(\mathscr{A}_{0}^{*}, \mathscr{A}_{1}^{*}, \ldots, \mathscr{A}_{J}^{*}\right)$, сопряженного с оператором $\mathscr{A}=$ $\left(\mathscr{A}_{0}, \mathscr{A}_{1}, \ldots, \mathscr{A}_{J}\right)$, совпадает с пространством (4.1).

В рассматриваемой ситуации условия сопряжения упрощаются, поскольку $d=d_{0}$, представления (3.6) и (3.13) неразличимы и оба проектора $\mathfrak{P}^{j}$ и $\mathfrak{p}^{j}$ действуют в пространстве $\mathbb{R}^{n}$. Обобщенная формула Грина (4.6) обеспечивает следующее утверждение.

ПредЛОЖениЕ 6.1. Сужение $\mathscr{A}_{j}^{*}$ на подпространство столбиов $\mathbf{u}^{j} \in \mathfrak{H}$, подчиненных условиям сопряжения (4.5), является самосопряженным расширением оператора $\mathscr{A}$.

Если взять $\mathfrak{P}^{1}=\cdots=\mathfrak{P}^{J}=0$, т. е. устранить условие $(4.5)_{1}$, превратить $(4.5)_{3}$ в $\pi_{-}^{j} \mathbf{u}=0$ и тем самым перейти к «классическому» решению $(2.14)$, то оставшиеся условия (4.5) 2,4 вместе с (2.10) породят смешанные краевые задачи для систем (2.8), никак не взаимодействующие с задачей (2.6). Предложение 6.1 
не описывает ${ }^{1)}$ все возможные расширения оператора $\mathscr{A}$, например, из-за того, что условия (4.5) отвергают «дальнодействие», при котором векторы $u^{j}$ и $u^{k}$ для $j \neq k$ связываются напрямую. Тем не менее можно проверить, что для самосопряженного расширения с областью определения, содержащейся в пространстве Н из (2.14), компонента $\mathscr{A}_{0}$ оператора $\mathscr{A}$ расширяется по Фридрихсу, что отрицает какое-либо взаимодействие тела $\Omega$ с отростками $\Upsilon^{j}$.

ЗАмЕчАниЕ 6.1. В случае произвольного линеала полиномов (2.4) применение самосопряженных расширений усложняется. Во-первых, при $\varkappa \geqslant 1$ пространство $\mathfrak{D}$ не вкладывается в $L_{2}(\Omega)^{n}$, однако можно воспользоваться подходом, развитым в [21] и имеющим дело с расширениями симметрических операторов $\mathfrak{r}^{-2 \beta} L^{o}$ в весовых классах $V_{\beta}^{0}(\Omega)^{n}$. Во-вторых, при различающихся порядках $t_{j}+t_{k}$ элементов матричного дифференциального оператора $(2.9)$, эллиптического в смысле Дуглиса-Ниренберга, замыкание в $L_{2}(\Upsilon)^{T}$ производится просто лишь при блочно-диагональной матрице $A^{j}$ (см. пример 2.1(1)) и распадении (2.8) на несколько систем, эллиптических по Петровскому. Впрочем, существуют ситуации, в которых возможен переход к краевой задаче для интегро-дифференциального оператора $H^{2}(\Upsilon)^{\tau} \rightarrow L_{2}(\Upsilon)^{\tau}$ (подобное сведение систематически применялось в [14] при изучении спектральных задач в теории стержней). Предположим, что $\mathfrak{P}^{j}$ проецирует на подпространство $\mathbb{R}^{\tau}$ столбцов коэффициентов $\mathbf{a}_{i}^{j}$ при постоянных $p_{(j)}^{1,0}, \ldots, p_{(j)}^{\tau, 0}$ из базиса $\left\{p_{(j)}^{k, i}\right\}$, для которых $t_{1}=\cdots=t_{\tau}=1$. Пусть и оператор $\mathfrak{s}^{j} \mathfrak{p}^{j}$ действует из $\mathbb{R}^{d_{0}}$ в $\mathbb{R}^{\tau}$. Тогда при помощи $(4.5)_{2}$ и $(4.5)_{1}$ определяются все граничные данные в точке $z^{j}=0$ для компонент $u_{k}^{j}$, которым отвечают порядки $t_{k} \geqslant 2$, и, следовательно, эти компоненты можно исключить из системы (2.8).

\section{§7. Интерпретации}

Обсудим две задачи математической физики, упомянутые в примере 2.1. Обозначим через $\omega^{j} \subset \mathbb{R}^{2}$ области, ограниченные простыми гладкими контурами $\partial \omega^{j}$, и введем тонкие цилиндры

$$
Q^{j} \varepsilon=\left\{x: \eta^{j}:=\varepsilon^{-1} y^{j}=\left(\varepsilon^{-1} y_{1}^{j}, \varepsilon^{-1} y_{2}^{j}\right) \in \omega^{j}, z^{j} \in\left(-\varepsilon \lambda_{j}, l_{j}\right)\right\}, \quad j=1, \ldots, J,
$$

где $\varepsilon \in\left(0, \varepsilon_{0}\right]$ - малый параметр, $\varepsilon_{0}>0$, и $y^{j}$ - декартовы координаты в плоскостях, перпендикулярных лучу $\Lambda^{j}$. Для простоты предполагаем, что область $\Omega$ выпуклая, лучи $\Lambda^{j}$ трансверсальны ее границе, а размер $\lambda_{j}>0$ выбран так, чтобы при $z=-\varepsilon \lambda_{j}$ сечение $\omega_{\varepsilon}^{j}(z)=\left\{x: \eta^{j} \in \omega^{j}, z^{j}=z\right\}$ лежало внутри $\Omega$. На сочленении $\Omega(\varepsilon)$ тела $\Omega$ со стержнями $G_{\varepsilon}^{j}=Q_{\varepsilon}^{j} \backslash \bar{\Omega}$ рассмотрим сначала смешанную краевую задачу с оператором Лапласа $\Delta_{x}$

$$
\begin{gathered}
-\Delta_{x} U^{0}=F^{0} \quad \text { в } \Omega, \quad-\mu \Delta_{x} U^{j}=F^{j} \quad \text { в } G_{\varepsilon}^{j}, \\
U^{0}=U^{j}, \quad \partial_{\nu} U^{0}=\mu \partial_{\nu} U^{j} \quad \text { на } \Gamma_{\varepsilon}^{j}=Q_{\varepsilon}^{j} \cap \partial \Omega, \\
\partial_{\nu} U:=\nu^{\top} \nabla_{x} U=0 \quad \text { на } \partial \Omega(\varepsilon) \backslash\left(\Gamma_{\varepsilon}^{1} \cup \cdots \cup \Gamma_{\varepsilon}^{J}\right), \\
U^{j}=0 \quad \text { на } \omega_{\varepsilon}^{j}\left(l_{j}\right), \quad j=1, \ldots, J .
\end{gathered}
$$

\footnotetext{
1)Это нетрудно сделать на основе общих результатов $[20,22,23]$.
} 
Параметр $\mu>0$, большой или малый, соотнесем с «толщиной» стержней $\varepsilon$ :

$$
\mu=\mu_{0} \varepsilon^{s}, \quad s \in \mathbb{R} .
$$

В соответствии с [10] (см. также [9,25] для случая $\mu=1$ ) согласованные асимптотические анзацы для сужений $F^{q}$ и $U^{q}$ правой части $F$ и решения $U$ выглядят так:

$$
\begin{gathered}
F^{0}(x)=f^{0}(x), \quad F^{j}(\varepsilon, x)=\varepsilon^{s+\mathbf{s}_{j}}\left\{\left(\operatorname{mes}_{2} \omega_{j}\right)^{-1} f^{j}\left(z^{j}\right)+f_{\perp}^{j}\left(\eta^{j}, z^{j}\right)\right\}, \\
U^{0}(\varepsilon, x) \sim \varepsilon^{\mathbf{s}_{0}} c^{0}+u_{\perp}^{0}(x)+\ldots, \quad U^{j}(\varepsilon, x) \sim \varepsilon^{\mathbf{s}_{j}} u^{j}\left(z^{j}\right)+\ldots,
\end{gathered}
$$

где $\mathbf{s}_{0}=-1-(1+s)_{+}, \mathbf{s}_{j}=-1-s / 2$ и

$$
t_{+}=\frac{1}{2}(t+|t|), \quad \int_{\omega_{j}} f_{\perp}^{j}\left(\eta^{j}, z^{j}\right) d \eta^{j}=0, \quad \int_{\Omega} u_{\perp}^{0}(x) d x=0 .
$$

Показатель $\mathbf{s}_{j}$ подобран так, чтобы интеграл Дирихле, вычисленный на стержне по анзацу из $(7.3)_{2}$, имел порядок $\varepsilon^{0}=1$. Из-за того что предельная задача Неймана в $\Omega$ не является однозначно разрешимой, в $(7.3)_{2}$ присутствует большой множитель $\varepsilon^{\mathbf{s}_{0}}$ при постоянной $c^{0}$ и составляющая $u_{\perp}^{0}$, а значит, и сумма $u^{0}=\varepsilon^{\mathbf{s}_{0}} c^{0}+u_{\perp}^{0}$ обладают сингулярностями в точках $\stackrel{P}{j}^{j}$ (см. [10] и $[9,11])$. Подставив $(7.3)^{\perp}$ в $(7.1)_{1,3,4}$, обнаруживаем, что компоненты столбца $\mathbf{u}=\left(u^{0}, u^{1}, \ldots, u^{J}\right)^{\top}$ удовлетворяют соотношениям

$$
\begin{gathered}
-\Delta_{x} u^{0}(x)=f^{0}(x), \quad x \in \Omega, \partial_{\nu} u^{0}=0, x \in \partial \Omega \backslash \mathbf{P}, \\
-\mu_{0} \operatorname{mes}_{2} \omega_{j} \partial_{z^{j}}^{2} u^{j}\left(z^{j}\right)=f^{j}\left(z^{j}\right), \quad z^{j} \in \Upsilon^{j}, u^{j}\left(l_{j}\right)=0 .
\end{gathered}
$$

Подчеркнем, что постоянное слагаемое исчезает из $(7.5)_{1}$. Справедливо представление (3.13), где $\mathbf{b}^{j}=\pi_{-}^{j} \mathbf{u} \in \mathbb{R}$, a $\mathfrak{G}^{j}$ - обобщенная функция Грина [19], т. е. имеющее нулевое среднее решение задачи

$$
-\Delta_{x} \mathfrak{G}^{j}(x)=-\left(\operatorname{mes}_{3} \Omega\right)^{-1}, \quad x \in \Omega, \quad \partial_{\nu} \mathfrak{G}^{j}(x)=\delta\left(x-P^{j}\right), \quad x \in \partial \Omega .
$$

Сингулярностей $\left(2 \pi r_{j}\right)^{-1} \mathbf{b}^{j}$ у решения исходной задачи (7.1) нет, и поэтому в зонах присоединения стержней к телу возникает явление пограничного слоя, описываемое в растянутых координатах $\eta^{j}$ и $\zeta^{j}=\varepsilon^{-1} z^{j}$ при помощи решений задач в полупространстве $\mathbb{R}_{j-}^{3}=\left\{\xi^{j}=\left(\eta^{j}, \zeta^{j}\right): \nu\left(P^{j}\right)^{\top} \xi^{j}<0\right\}$, в усеченном цилиндре $Q_{+}^{j}=\left\{\xi^{j} \in \omega^{j} \times \mathbb{R}: \nu\left(P^{j}\right)^{\top} \xi^{j}<0\right\}$ или на их объединении (подробности см. в [9-11]). Далее потребуется только гармонический емкостной потенциал $Y^{j}(\xi)=\mathfrak{c}_{j}\left(4 \pi\left|\xi^{j}\right|\right)^{-1}+O\left(\left|\xi^{j}\right|^{-2}\right)$ для диска $B^{j}=\partial \mathbb{R}_{j-}^{3} \cap \partial Q_{+}^{j}$ в $\mathbb{R}^{3}$ и соответствующая емкость $(4 \pi)^{-1} \mathfrak{c}_{j}>0$.

В качестве асимптотики решения $U^{0}(\varepsilon, x)$ вблизи точки $P^{j}$ берется сумма $\mathbf{a}^{j}+2 \mathbf{b}^{j} \mathfrak{c}_{j}^{-1} \varepsilon^{-1} Y^{j}(\xi)$, принимающая значение $\mathbf{a}^{j}+2 \mathbf{b}^{j} \mathfrak{c}_{j}^{-1} \varepsilon^{-1}$ на $\Gamma_{\varepsilon}^{j}$; здесь $\mathbf{a}^{j}=$ $\pi_{+}^{j} \mathbf{u}$. Сравнивая это значение с анзацем $(7.3)_{2}$ для $U^{j}(\varepsilon, x)$, выводим первое условие сопряжения

$$
\pi_{+}^{j} \mathbf{u}+2 \mathfrak{c}_{j}^{-1} \varepsilon^{-1} \pi_{-}^{j} \mathbf{u}=\varepsilon^{\mathbf{s}_{j}}\left(\pi^{j} \mathbf{u}\right)(0)
$$

Второе условие сопряжения получается в результате вычисления потоков через поверхность $\Gamma_{\varepsilon}^{j}$ и принимает вид $\mathbf{b}^{j}=\mu_{0} \varepsilon^{s} \operatorname{mes}_{2} \omega_{\varepsilon}^{j} \varepsilon^{\mathbf{s}_{j}} \partial_{z^{j}} u^{j}(0)$, т. е. в соответствии с $(7.5)_{2}$

$$
\varepsilon^{\mathbf{s}_{j}} \pi_{-}^{j} \mathbf{u}=N^{j}\left(\partial_{z^{j}}\right)\left(\pi^{j} \mathbf{u}\right)(0)
$$


Если положить $\mathfrak{P}^{j}=\mathfrak{p}^{j}=\mathbb{I}, \mathfrak{S}^{j}=\mathfrak{c}_{j}^{-1} \varepsilon^{-1}$ и $\mathfrak{s}^{j}=\varepsilon^{\mathbf{s}_{j}}$, то соотношения (4.5) превратятся в (7.6), (7.7). Само по себе такое наблюдение малоинтересно, однако можно убедиться в том, что на всем диапазоне изменения параметра $s$ (или $\mu$ ) решение задачи (7.5)-(7.7) дает приемлемую аппроксимацию решения задачи (7.1). А именно, повторив с некоторыми изменениями проверку оценок асимптотических остатков в [10], приходим к следующему утверждению.

Tеорема 7.1. Пусть $f^{0} \in L^{2}(\Omega), f^{j} \in L^{2}\left(\Upsilon^{j}\right)$, a $\partial \Omega \Omega f_{\perp}^{j} \in L^{2}\left(\omega^{j} \times \Upsilon^{j}\right)$ выполняется условие ортогональности из (7.4). Тогда решения $U \in H^{1}(\Omega(\varepsilon))$ $u \mathbf{u} \in \mathfrak{H}$ задач (7.1) и (7.5)-(7.7) связаны соотношением

$$
\begin{aligned}
\varepsilon^{-\mathbf{s}_{0} / 2}\left|\bar{U}^{0}-\bar{u}^{0}\right|+\left\|\left(U^{0}-\bar{U}^{0}\right)-\left(u^{0}-\bar{u}^{0}\right) ; V_{1}^{1}(\Omega)\right\| \\
+\varepsilon^{s / 2} \sum_{j=1}^{J}\left\|U^{j}-\varepsilon^{\mathbf{s}_{j}} u^{j} ; H^{1}\left(G_{\varepsilon}^{j}\right)\right\| \leqslant c \varepsilon^{\left(1+\mathbf{s}_{0}\right) / 2} \mathscr{N}(\varepsilon),
\end{aligned}
$$

где чертой обозначено среднее по $\Omega$, постоянная с не зависит от параметров $\varepsilon \in\left(0, \varepsilon_{0}\right], s \in \mathbb{R}$ и правых частей $(7.3)_{1}$, a $\mathscr{N}(\varepsilon)$ - сумма норм функиий $f^{0}$, $\varepsilon^{s+\mathbf{s}_{j}} f^{j} u \varepsilon^{s+\mathbf{s}_{j}} f_{\perp}^{j}$ в указанных пространствах.

Задача (7.5)-(7.7) по-прежнему содержит малый параметр $\varepsilon$, однако ее следует интерпретировать как регулярно возмущенную. Построение асимптотики ее решения, которое разлагается в сходящийся ряд, несложно. В частности, простейший анализ условий сопряжения (7.6), (7.7) показывает, что главный член асимптотики, составляющей $u^{0}$, имеет вид $(7.3)_{2}$.

ЗАмЕчАниЕ 7.1. Если $s \geqslant 0$, то среди предельных задач для (7.1) (см. [10]) нет смешанной краевой задачи в полупространстве $\mathbb{R}_{j-}^{3}$ с условиями Дирихле на диске $B^{j}$ и условиями Неймана на $\partial \mathbb{R}_{j-}^{3} \backslash \bar{B}^{j}$, т. е. емкостной потенциал $Y^{j}$ и емкость $(4 \pi)^{-1} \mathfrak{c}_{j}$ не фигурируют в асимптотике. Однако оказывается, что погрешности одномерной модели $(7.5)_{2}$ стержня больше, чем невязка, привносимая потенциалом, и поэтому слагаемое $2 \mathfrak{c}_{j}^{-1} \varepsilon^{-1} \pi_{-}^{j} \mathbf{u}$ можно оставить в (7.6) и при $s \geqslant 0$. Подчеркнем, что величина $2 \mathbf{b}^{j} \mathfrak{c}_{j}^{-1} \varepsilon^{-1} Y^{j}\left(\xi^{j}\right)$ отсутствует в левой части неравенства (7.8) из-за весовых множителей в $V_{1}^{1}(\Omega)$-норме, малых около точек $P^{j}$ (см. (3.1)).

Аналогичное моделирование можно провести и для задачи теории упругости, упомянутой в примере 2.1(1). Ограничимся несколькими замечаниями, поскольку соответствующий анализ становится более громоздким из-за увеличения размерности линеала $\mathscr{P}$, которая влечет за собой усложнение строения базиса (4.4):

$$
p_{(j)}^{k, 0}=e_{k}^{(j)}, \quad p_{(j)}^{k, 1}=z^{j} e_{k}^{(j)} \quad(k=1,2), \quad p_{(j)}^{3,0}=e_{3}^{(j)}, \quad p_{(j)}^{4,0}=y_{2}^{j} e_{1}^{(j)}-y_{1}^{j} e_{2}^{(j)},
$$

и структуры асимптотического анзаца на стержнях (см. $[12,14,28])$ :

$$
U^{j}(\varepsilon, x) \sim \varepsilon^{\mathbf{s}_{j}} \sum_{j=1}^{J} \varepsilon^{t_{k}} \sum_{i=0}^{t_{k}-1} \varepsilon^{i} p_{(j)}^{k, i}\left(\eta^{j}, 0\right) \frac{1}{i !} \partial_{z^{j}}^{i} u_{k}^{j}\left(z^{j}\right) .
$$

Здесь $e_{k}^{(j)}$ и $e_{3}^{(j)}-$ орты осей $y_{k}^{j}$ и $z^{j}$. Поперечные смещения $u_{1}^{j}\left(z^{j}\right), u_{2}^{j}\left(z^{j}\right)$ и продольное смещение $u_{3}^{j}\left(z^{j}\right)$, а также закручивание стержня $u_{4}^{j}\left(z^{j}\right)$ входят в 
правую часть соотношения (7.10) с разными множителями $\varepsilon^{\mathbf{s}_{j}-2}$ и $\varepsilon^{\mathbf{s}_{j}-1}$ соответственно, что отражает житейски очевидный факт: упругий стержень легче изогнуть, чем растянуть или скрутить. Поэтому в механике выделяются конструкции, в которых стержневые элементы «работают только на растяжение». Для условий (4.5) это означает, что $\mathfrak{P}^{j}$ и $\mathfrak{p}^{j}$ проецируют на подпространства столбцов $\mathbf{a}^{j}$, у которых ненулевым может быть только коэффициент $\mathbf{a}_{3}^{j}$ при полиноме $p_{(j)}^{3,0}=e_{3}^{(j)}($ см. (4.4)). В силу следствия 4.1 обоснованное требование однозначной разрешимости задачи с подобными условиями сопряжения формулируется так:

$3^{\circ}$ линейная оболочка столбцов $\mathbf{p}\left(P^{j}\right)^{\top} \nu\left(p^{j}\right), j=1, \ldots, J$, совпадает $с \mathbb{R}^{6}$.

Другая характерная конфигурация стержней связана с возможностью игнорировать передачу поворотов от тела стержням - проекторы $\mathfrak{P}^{j}$ и $\mathfrak{p}^{j}$ действуют в подпространство столбцов с элементами $\mathbf{a}_{k}^{j}=0$ при $k \neq 1,3,5$ (исключительными являются жесткие поступательные смещения; ср. (7.9) с (4.4)). Теперь необходимые условия из следствия 4.1 равносильны тому, что

$4^{\circ}$ линейная оболочка столбиов $\mathbf{p}\left(P^{1}\right)^{\top} \ldots \mathbf{p}\left(P^{J}\right)^{\top}$ cовпадает $c \mathbb{R}^{6}$.

В $3^{\circ}$ возникает ограничение $J \geqslant 6$, а в $4^{\circ}$ - ограничение $J \geqslant 3$; если $J<3$, упрощенные модели не годятся. Требования $3^{\circ}$ и $4^{\circ}$ уже возникали в [24] при выводе асимптотически точных неравенств Корна, а в [11,26,27] - при асимптотическом анализе упругих сочленений, и в обоих случаях обеспечивали специфические свойства исследуемых объектов.

ЗАмЕчАниЕ 7.2. Как установлено в $[12,28]$, структура (7.10) начальных членов асимптотики сохраняется и для общих эллиптических задач с полиномиальным свойством на тонких областях. Разложение функций $u_{k}^{j}$ в ряды Маклорена формирует справа в (7.10) полиномы $p_{(j)}^{k, i}\left(y^{j}, z^{j}\right)$, фигурирующие в представлении (3.6) для $u^{0}$. Иными словами, условия сопряжения $(4.5)_{1,4}$ обеспечивают сращивание асимптотических разложений на теле и стержнях, а операторы $\mathfrak{s}^{j}$ и $\mathfrak{S}^{j}$ служат для выравнивания порядков относительно $\varepsilon$ и учета эффектов пограничного слоя. Условия сопряжения $(4.5)_{2,3}$, парные для $(4.5)_{1,4}$ ввиду обобщенной формулы Грина, выражают равенство потоков разнообразных физических полей через поверхность соединения $\Gamma_{\varepsilon}^{j}$.

\section{ЛитеРАТУРА}

1. Lumer $G$. Connecting of local operators and evolution equation on network. In: Lect. Notes in Math., Vol. 787, Springer-Verlag, 1980, pp. 219-234.

2. Павлов Б. С., Фаддеев М. Д. Модель свободных электронов и теория рассеяния. Теорет. и матем. физика, 55, №2, 257-269 (1983).

3. Roth J. P. Le spectre du laplacien sur un graphe. In: Lect. Notes in Math., Vol. 1096, Springer-Verlag, 1984, pp. 521-539.

4. Пенкин О. М., Покорный Ю. В. О некоторых качественных свойствах уравнений на одномерном клеточном комплексе. Матем. заметки, 64, №5, 777-780 (1996).

5. Naimark K., Solomyak M. Eigenvalue estimates for the weighted laplacian on metric trees. Proc. Lond. Math. Soc., 80, No. 3, 690-724 (2000).

6. Жиков B. В. Усреднение задач теории упругости на сингулярных структурах. Изв. РАН, сер. матем., 66, № 2, 81-148 (2002). 
7. Назаров C. А., Пламеневский Б. А. Обобщенная формула Грина для эллиптических задач в областях с ребрами. Проблемы матем. анализа, вып. 13, Изд-во СПбГУ, СПб, 1992, с. 106-147.

8. Назаров C. А., Пламеневский Б. А. Эллиптические задачи в областях с кусочно гладкой границей. Наука, М., 1991; English transl.: Nazarov S. A., Plamenevsky B. A. Elliptic problems in domains with piecewise smooth boundaries. Walter de Gruyter, Berlin-New York, 1994.

9. Kozlov V. A., Maz'ya V. G., Movchan A. B. Asymptotic analysis of a mixed boundary value problem in a multistructure. Asymptotic Analysis, 8, 105-143 (1994).

10. Назаров $C$. A. Соединения сингулярно вырождающихся областей различных предельных размерностей. 1. Труды семинара им. И. Г. Петровского, вып. 18, Изд-во МГУ, М., 1995, с. 3-78; 2. ibid, вып. 20, Изд-во МГУ, М., 1997, с. 155-195.

11. Kozlov V. A., Maz'ya V. G., Movchan A. B. Asymptotic analysis of fields in multistructures. Clarendon Press, Oxford, 1999.

12. Назаров C. A. Полиномиальное свойство самосопряженных эллиптических краевых задач и алгебраическое описание их атрибутов. УМН, 54, вып. 5, 77-142 (1999).

13. Nečas J. Les méthodes directes in théorie des équations elliptiques. Masson-Academia, Paris-Prague, 1967.

14. Назаров C. A. Асимптотическая теория тонких пластин и стержней. Понижение размерности и интегральные оценки. Научная книга, Новосибирск, 2002.

15. Кондратъев B. A. Краевые задачи для эллиптических уравнений в областях с коническими или угловыми точками. Труды ММО, 16, 219-292 (1963).

16. Мазъя В. Г., Пламеневский Б. А. О коэффициентах в асимптотике решений эллиптических краевых задач в области с коническими точками. Math. Nachr., 76, 29-60 (1977).

17. Назаров $C$. А., Пламеневский Б. А. Эллиптические задачи с условиями излучения на ребрах границы. Матем. сб., 183, вып. 10, 13-44 (1992).

18. Pazy A. Asymptotic expansions of solutions of ordinary differential equations in Hilbert space. Arch. Rat. Mech. Anal., 24, No. 2, 193-218 (1967).

19. Смирнов В. И. Курс высшей математики, т. 2. Наука, М., 1967.

20. Павлов Б. С. Теория расширений и явно решаемые модели. УМН, 42, вып. 6, 99-132 (1987).

21. Назаров C. A. Самосопряженные расширения оператора задачи Дирихле в весовых функциональных пространствах. Матем. сб., 137, вып. 2, 224-241 (1988).

22. Рофе-Бекетов $\Phi$. С. Самосопряженные расширения дифференциальных операторов в пространстве вектор-функций. ДАН СССР, 184, 1034-1037 (1969).

23. Горбачук В. И., Горбачук М. Л. Граничные задачи для дифференциально-операторных уравнений. Наукова думка, Киев, 1984.

24. Nazarov S. A. Korn's inequalities for junctions of spatial bodies and thin rods. Math. Methods Appl. Sci., 20, No. 3, 219-243 (1997).

25. Kozlov V. A., Maz'ya V. G., Movchan A. B. Asymptotic representation of elastic fields in a multi-structure. Asymptotic Analysis, 11, 343-415 (1995).

26. Назаров C. A. Асимптотика решений задачи теории упругости для трехмерного тела с тонкими отростками. Докл. РАН, 352, No. 4, 458-461 (1997).

27. Kozlov V. A., Maz'ya V. G., Movchan A. B. Fields in non-degenerate 1D-3D elastic multi-structures. Quart. J. Mech. Appl. Math., 54, 177-212 (2001).

28. Назаров C. A. Общая схема осреднения самосопряженных эллиптических систем в многомерных областях, в том числе тонких. Алгебра и анализ, 7, вып. 5, 1-92 (1995).

Институт проблем машиноведения РАН email: serna@snark.ipme.ru

Поступило в редакцию 18 ноября 2003 г. 